\title{
Meroxest improves the prognosis of immunocompetent C57BL/6 mice with allografts of E0771 mouse breast tumor cells
}

\author{
Esther Carrasco ${ }^{1,2}$, Jose Manuel Garrido ${ }^{3}$, Pablo Juan Álvarez ${ }^{1}$, Enrique Álvarez-Manzaneda4, \\ Rachid Chahboun ${ }^{4}$, Ibtissam Messouri' ${ }^{4}$, Consolación Melguizo을 Antonia Aránega ${ }^{1}$, \\ Fernando Rodríguez-Serrano ${ }^{1}$
}

\begin{abstract}
${ }^{1}$ Institute of Biopathology and Regenerative Medicine (IBIMER), University of Granada, Granada, Spain

${ }^{2} \mathrm{PhD}$ Program in Biomedicine, University of Granada, Granada, Spain

${ }^{3}$ Department of Cardiovascular Surgery, Virgen de las Nieves Hospital, Granada, Spain ${ }^{4}$ Department of Organic Chemistry, Institute of Biotechnology, University of Granada, Granada, Spain
\end{abstract}

Submitted: 28 July 2014

Accepted: 7 September 2014

Arch Med Sci 2016; 12, 5: 919-927

DOI: $10.5114 /$ aoms.2014.45442

Copyright $\odot 2016$ Termedia \& Banach

\section{Abstract}

Introduction: Recently, we have reported the antitumor properties of a new family of synthetic merosesquiterpenes, among which meroxest is highlighted, since it has high activity and specificity for $\mathrm{ER}^{+}$breast cancer cells. In this paper, we characterize allografts of ER+E0771 mouse breast tumor cells in immunocompetent $\mathrm{C} 57 \mathrm{BL} / 6$ mice, and also analyze the effect of meroxest on the prognosis of the disease.

Material and methods: Twenty female C57BL/6 mice were injected with $10^{6}$ E0771 cells. Once the tumors reached the appropriate size, the mice were divided into two groups, one control and another treated orally with $15 \mathrm{mg}$ / $\mathrm{kg}$ of meroxest. After 20 days, tumor samples were taken for histopathological study and for determination of the expression of the prognostic markers Ki67 and vascular endothelial growth factor (VEGF) by immunofluorescence. Results: In sections stained with hematoxylin-eosin, we observed that tumors have a well-defined capsule enclosing E0771 tumor cells. The central area of tumors contains necrotic regions with leukocyte infiltration. Meroxest treatment significantly reduces tumor size $(68 \%, p<0.05)$, induces changes in its structure, decreases the degree of leukocyte infiltration, and significantly reduces the expression of Ki67 (33\%, $p<0.05)$ and VEGF $(82 \%, p<0.05)$.

Conclusions: Meroxest improves the prognosis of mice since it reduces leukocyte infiltration, and decreases the expression of Ki67 and VEGF markers. Consequently, the merosesquiterpene could become a useful antiangiogenic drug in the treatment of breast cancer. These results encourage us to deepen the study of meroxest, in order to find more evidence that supports the convenience of its evaluation in a clinical study or trial.

Key words: breast cancer, in vivo, Ki67, merosesquiterpene, vascular endothelial growth factor.

\section{Introduction}

Breast cancer is the most common cancer in women worldwide [1], and in the past few decades, the incidence has increased [2]. Despite

\author{
Corresponding author \\ Fernando Rodríguez-Serrano \\ Institute of Biopathology \\ and Regenerative Medicine \\ Biomedical Research Centre \\ Avd. del Conocimiento \\ s/n. 18100 Armilla \\ Granada, Spain \\ Phone: + 34958248826 \\ E-mail: fernrs@ugr.es
}


advances in detection and targeted therapies, it remains the first cause of death by cancer in females [3]. In general, oncogenic transformation is the result of alterations in genes associated with DNA repair, tumor suppression and/or genes involved in the cell cycle and apoptosis [4]. Clinically, this disease is highly heterogeneous, with several subtypes distinguished, differing in their biological characteristics and pathological behavior. This is associated with differences in prognosis and treatment response $[5,6]$. The use of immunohistochemistry and gene expression studies has allowed the classification of breast cancer in at least five molecular subtypes: luminal A, luminal B, HER2, and the triple negative subtypes, basal-like and claudin-low. This classification is established primarily depending on the presence of estrogen (ER) and/or progesterone (PR) receptors, and human epidermal growth factor receptor-2 (HER2). Among them, luminal A subtype is the most prevalent, characterized by ER and/or PR expression, but not HER2 amplification (ER+, $\mathrm{PR}^{+/}, \mathrm{HER2} 2^{-}$[7-9].

Breast cancer is currently treated with conservative surgery, adjuvant and/or neoadjuvant chemotherapy, radiotherapy, and hormone therapy, as appropriate. However, there is an elevated rate of patients who relapse with metastatic disease [10-12]. Therefore, we need to develop new treatments aimed at being more effective and selective, and that contribute to improving the prognosis and survival of patients.

Throughout the entire history of man, organisms of different nature have been used to alleviate all kinds of diseases, especially medicinal plants [13]. With technological development, compounds with different biological activity were purified from such organisms, including many widely administered "standard" chemotherapeutics such as paclitaxel, vinblastine and vincristine, among others [14].

Natural products of mixed biosynthetic origin (polyketide-terpenoid) containing a sesquiterpene unit joined to a phenolic or quinone moiety are

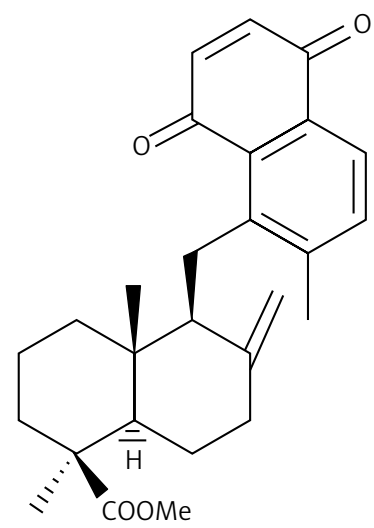

Figure 1. Chemical structure of meroxest generally named "merosesquiterpenes" [15]. The most important metabolites of this family are the compounds bearing a bicyclic terpene (drimane) moiety, mainly due to their potent biological activities. As an example we can highlight the drimenyl phenols wiedendiol $A$ and wiedendiol $B$, which are inhibitors of the cholesteryl ester transfer protein (CETP) inhibitors, isolated from the marine sponge Xestospongia wiedenmayeri [16, 17]. Other examples within the drimenyl quinones are the antitumor tauranin, isolated from Phyllosticta spinarum, a fungal strain endophytic in Platycladus orientalis [18], which also inhibits cholesterol biosynthesis; the anti-HIV (p)-hyatellaquinone (4), found in the sponge Hyatella intestinalis [19, 20]; and the recently reported discomycete metabolite (-)-F-12509 A, with sphingosine kinase inhibitory activity [21].

We have recently reported the antitumor properties of a new family of synthetic merosesquiterpenes. These compounds, whose synthesis and effects are protected under international patents WO/2009/112622 and WO/2010/076358, have cytotoxic activity against human breast, colon, and lung tumor cells. However, they have shown greater specificity against breast cells. Among them, compound 13 was highlighted, henceforth referred to as meroxest (Figure 1). It has elevated activity and specificity against the luminal breast cancer cell MCF-7. We proved that potent antitumor activity of meroxest against MCF-7 was mediated by the induction of oxidative stress; cell cycle arrest in $\mathrm{G}_{0}-\mathrm{G}_{1}$ phase accompanied by downregulation of cyclin D1, pRb hypophosphorylation and increased expression of p27; and apoptosis associated with increased expression of p53 and poly (ADP-ribose) polymerase (PARP) fractioning. Moreover, meroxest appears to inhibit epithelial-mesenchymal transition, a process involved in metastasis and associated with poor prognosis. The in vitro results prompted us to assess its effect in vivo. For this purpose, we analyzed the effect of meroxest on tumor growth in immunocompetent C57BL/6 mice with allografts of E0771 mouse breast tumor cells (luminal subtype), and we found that meroxest markedly reduced the volume of tumors [22].

In this paper, we characterize the histopathology of allografts of E0771 mouse breast tumor cells in immunocompetent $\mathrm{C} 57 \mathrm{BL} / 6$ mice, and we also analyze the effect of meroxest on the prognosis of the disease, considering two markers widely used for this purpose, Ki67 and vascular endothelial growth factor (VEGF).

\section{Material and methods}

\section{Cell line and culture}

Mouse breast tumor line E0771 was supplied by the Cell Culture Service of the Scientific Instru- 
mentation Center (University of Granada). The cell line was cultured at $37^{\circ} \mathrm{C}$ in $5 \% \mathrm{CO}_{2}$ and $90 \%$ humidity with Dulbecco's modified Eagle medium (DMEM), supplemented with $10 \%$ heat-inactivated fetal bovine serum, $10 \mathrm{ml} / \mathrm{l}$ penicillin-streptomycin 100X, and $2 \mathrm{mM} \mathrm{L-glutamine.} \mathrm{Culture} \mathrm{media}$ and supplements were supplied by Sigma-Aldrich (St. Louis, MO).

\section{Subcutaneous allograft study}

Twenty female C57BL/6 mice weighing 25-30 g were purchased from Scientific Instrumentation Center of Granada University and were kept in a laminar flow cabinet in a room with a controlled environment $\left(37^{\circ} \mathrm{C}, 40-70 \%\right.$ relative humidity, $12 \mathrm{~h}$ light/dark cycle, and pathogen-free). The in vivo study was approved by the ethics committee of the University of Granada. An exponentially growing E0771 cell suspension containing $1 \times 10^{6}$ cells was injected subcutaneously in the right side of the mice. At 9 days, when the tumor volume reached $75 \mathrm{~mm}^{3}$, mice were randomly distributed into two groups, for oral treatment with vehicle alone (1\% methylcellulose) or with compound 13 at a concentration of $15 \mathrm{mg} / \mathrm{kg}$. The compound was administered on post-injection days 9, 12, 15, 18,21 , and 24.

\section{Histological analysis}

On day 42 after injection, mice were sacrificed and tissue samples obtained from tumor allografts were processed as described previously [23]. Briefly, samples were fixed in $10 \%$ neutral buffered formalin, embedded in paraffin, cut at $5 \mu \mathrm{m}$ thickness, and stained with hematoxylin and eosin for histological examination.

\section{Immunofluorescence analysis of Ki67 and VEGF expression}

Sections were deparaffinized in xylene and gradually hydrated through a graded series of ethanol solutions from $100 \%$ to $50 \%$ and $\mathrm{dd}_{2} \mathrm{O}$. For antigen retrieval, slides were placed in a container with $10 \mathrm{mM}$ sodium citrate buffer at $95^{\circ} \mathrm{C}$ for 10 min. Later, slides were washed in $\mathrm{ddH}_{2} \mathrm{O}$ and preincubated in a humidity box with $10 \%$ normal serum, $0.1 \%$ Tween and $0.1 \%$ triton in PBS for $30 \mathrm{~min}$ to block nonspecific labeling. Blocking solution was removed and primary antibody against Ki67 (sc-7846) or VEGF (sc-7269) was added and samples were incubated overnight at $4^{\circ} \mathrm{C}$. Antibody solution was removed and slides were washed with PBS-Tween $0.1 \%$ three times for 5 min each. Slides were incubated with secondary antibody, anti-goat (sc-2024) or anti-mouse (sc-2010), for $1 \mathrm{~h}$ at room temperature in a dark room and washed with PBS-Tween $0.1 \%$ three times for 5 min each. Following DAPI counterstaining, the tissue sections were examined and photographed with a fluorescence microscope (Leica DM IL LED Fluo). Total fluorescence measurements were performed taking 30 fields of vision from each slide and were analyzed using ImageJ software.

\section{Statistical analysis}

SPSS 14 for Windows (SPSS, Chicago, IL, USA) was used for the statistical analysis. Results were compared with Student's test, and $p<0.05$ was considered significant. Data were graphically represented using Microsoft Excel 2010 software (Microsoft Corporation).

\section{Results}

\section{Analysis of tumors}

We found that the rate of tumor formation using allografts of E0771 cells in immunocompetent C57BL/ 6 mice was very high, over $95 \%$ of cases. In Figure 2A, a mouse bearing a tumor can be observed, and panel $2 \mathrm{~B}$ shows photographs of tumors isolated from the sacrificed animals. The mean volume of control tumors was $12.14 \times 10^{3} \mathrm{~mm}^{3}$, whereas that of tumors treated with meroxest was $3.89 \times 10^{3} \mathrm{~mm}^{3}$. Macroscopically, we found that meroxest significantly reduces tumor size (Figure 2B1), and also that it induces changes in tumor structure, as compared to controls (Figure $2 \mathrm{~B} 2$ vs. 2B3). We found important differences in the texture of tumors, since controls showed a consistency similar to liver, while tumors of animals treated with meroxest presented a more compact and robust consistency, similar to cartilage tissue (Figure 2B3).

In sections stained with hematoxylin-eosin, we observed that the tumors of E0771 cells exhibited a well-defined capsule formed by normal tissue, enclosing a mass of tumor cells. The central area of the tumors contained necrotic centers with abundant debris and leukocyte infiltration (Figures $3 \mathrm{~A}$ and $\mathrm{B}$ ). Interestingly, we often found that the tumor cells were capable of invading the underlying muscle tissue (Figures $3 \mathrm{C}$ and D). Tumors from animals treated with meroxest shared the characteristics described for the control tumors, except that significantly less leukocyte infiltration was found in tumors from meroxest-treated mice (Figures $3 \mathrm{E}$ and $\mathrm{F}$ ).

\section{Immunofluorescence analysis of Ki67 and VEGF expression}

We analyzed the expression of the prognostic markers Ki67 and VEGF by immunofluorescence. We observed high expression of Ki67 in tumor cells of control animals. However, animals treated 
A

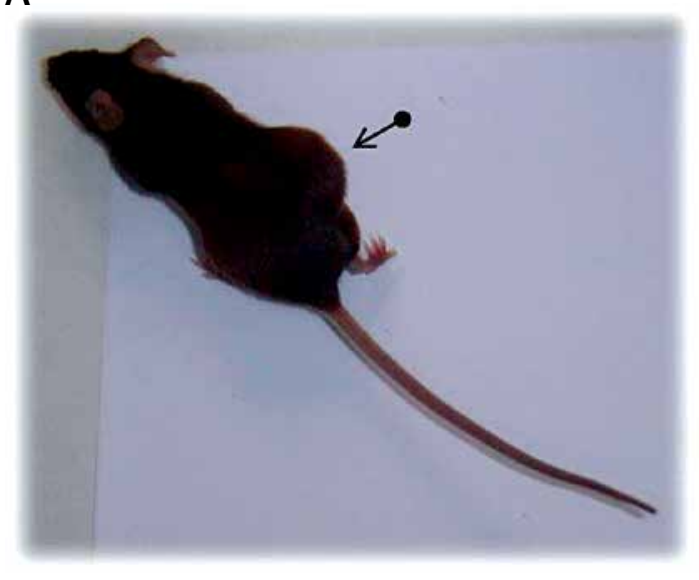

B

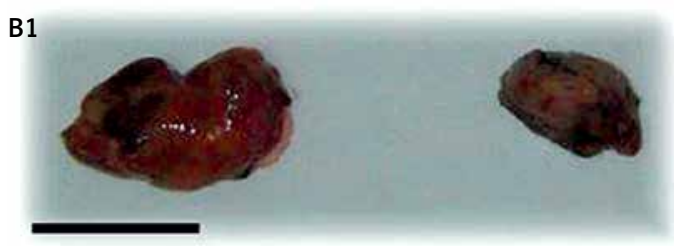

B2

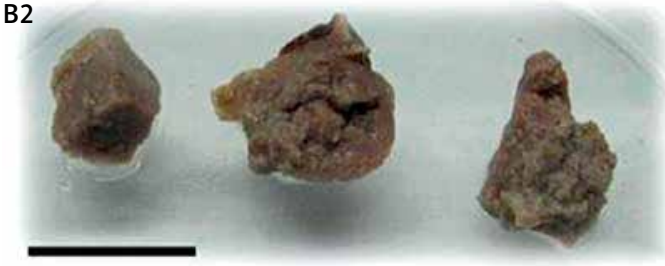

B3

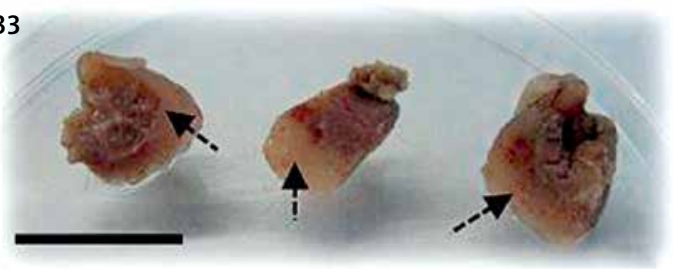

Figure 2. Allografts of E0771 cells in C57BL/6 mice. A - Mouse bearing a tumor (arrow). B - Samples of tumors: whole tumor from control (left) and from $15 \mathrm{mg} / \mathrm{kg}$ meroxest-treated animal (right) (B1). The mean volume of control tumors was $12.14 \times 103 \mathrm{~mm}^{3}$, whereas that of tumors treated with meroxest was $3.89 \times 103 \mathrm{~mm}^{3}$. Fragments from control tumors (B2) and from $15 \mathrm{mg} / \mathrm{kg}$ meroxest-treated tumors (B3). Arrows in B3 indicate areas with marked modifications in the structure of meroxest-treated tumors as compared to controls. Bars: $24 \mathrm{~mm}$ (B1) and $15 \mathrm{~mm}$ (B2 and B3)

with meroxest experienced a significant decrease in Ki67 expression of over 33\%. Similarly, expression of VEGF was significantly reduced by more than $80 \%$ in treated animals as compared with controls (Figures 4 and 5).

\section{Discussion}

Meroxest is a novel merosesquiterpene which was synthesized by Diels-Alder cycloaddition of the labdane diene trans-communic acid, highly abundant in Cupressus sempervirens. Previously, we reported that meroxest acts selectively against breast tumor cells through a mechanism that comprises the arrest of cells in $\mathrm{G}_{0}-\mathrm{G}_{1}$ phase, and the induction of oxidative stress and apoptosis. Furthermore, meroxest is capable of inhibiting the growth of tumors in vivo [22]. In this paper, we have characterized allografts of E0771 mouse breast tumor cells in immunocompetent C57BL/6 mice. Moreover, we have evaluated the effects of meroxest on tumor development, at both macroscopic and microscopic levels, and the expression of two important prognostic markers, Ki67 and VEGF. Ki67 is a classic proliferation marker widely used in clinical practice, and it is considered as an ideal marker for monitoring breast cancer [24]. By contrast, VEGF is a marker whose importance has been emphasized more recently, because of its involvement in an important process for tumor development, angiogenesis [25].

We have used E0771 murine breast cancer cells to induce syngeneic allografts in C56BL/C mice since it is an immunocompetent experimental model. We believe that this system provides translational results due to most breast cancer patients also being immunocompetent. It must be considered that an immunocompetent microenvironment is necessary for the study of cancer stem cells (CSCs) in vivo and the complex interaction between therapies targeting CSCS and the immune system $[26,27]$. This is very important, because according to the hypothesis of CSCs, these cells would be responsible for the origin and progression of cancer, as well as relapse and treatment failure [28]. Moreover, allografts of E0771 cells in C56BL/C mice are subcutaneous models with high invasiveness, a typical feature of human breast cancer $[26,29]$, as we have found in our samples. All these aspects, along with the high rate of tumor formation, make us believe that the experimental model chosen is suitable for the proposed study. However, there are few articles that refer to the use of the E0771 model in C57BL/6 mice, and we believe that its use should be more widespread.

We first analyzed the tumors macroscopically. We found important differences between tumors 

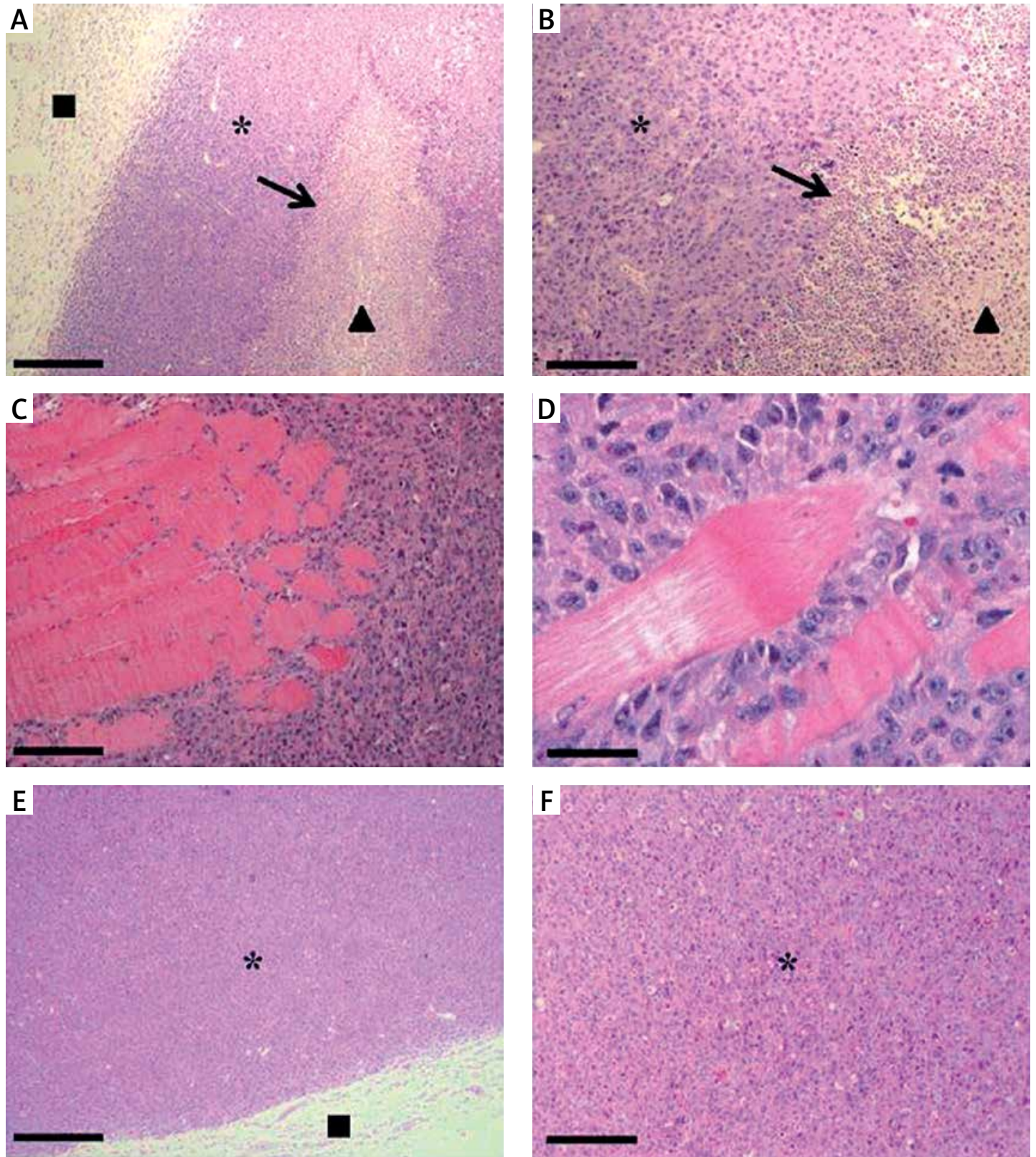

Figure 3. Histological analysis of E0771 allografts. A - Micrograph of a hematoxylin-eosin-stained control tissue section showing a peripheral capsule of normal tissue $(\boldsymbol{\square})$, enclosing tumor cells $\left(^{*}\right)$ and necrotic centers $(\boldsymbol{\Delta})$ with leukocyte infiltration (arrow). B - Micrograph with higher magnification which shows leukocyte infiltration at the edge of a necrotic center. C and D - E0771 tumor cells invading the underlying muscle tissue. E and F - Tumor sections from a $15 \mathrm{mg} / \mathrm{kg}$ meroxest-treated animal exhibiting less leukocyte infiltration than controls. Bars: $367.5 \mu \mathrm{m}$ (A, E), $147 \mu \mathrm{m}(\mathrm{B}, \mathrm{C}, \mathrm{F})$ and $36.75 \mu \mathrm{m}$ (D)

treated and untreated with meroxest, exhibiting in the former cartilage-like consistency, while the latter had a consistency similar to the liver. This aspect is of relevance in the anatomo-pathological description of tumors since it reflects structural and ultrastructural modifications. Microscopically, control tumors had necrotic centers with abundant leukocyte infiltration. This histopathological organization concurs with the single previous description of these tumors that we found in the literature, resembling medullary breast tumors [29]. Interestingly, tumors from animals treated with meroxest experienced a significant reduction of leukocyte infiltration. At present, tumor-associated inflammation is considered a hallmark of tumor development, and there is growing evidence of its clinical significance in different types of tumors [30]. Paradoxically, the presence of leukocyte infiltration is associated with a good or bad clinical outcome depending on the type of tumor [31]. In the case of breast cancer its role is still unclear, and there are even contradictory studies, which could be due to the heterogeneity of this cancer [32]. In $\mathrm{ER}^{+}$breast tumors, leukocyte 
Control

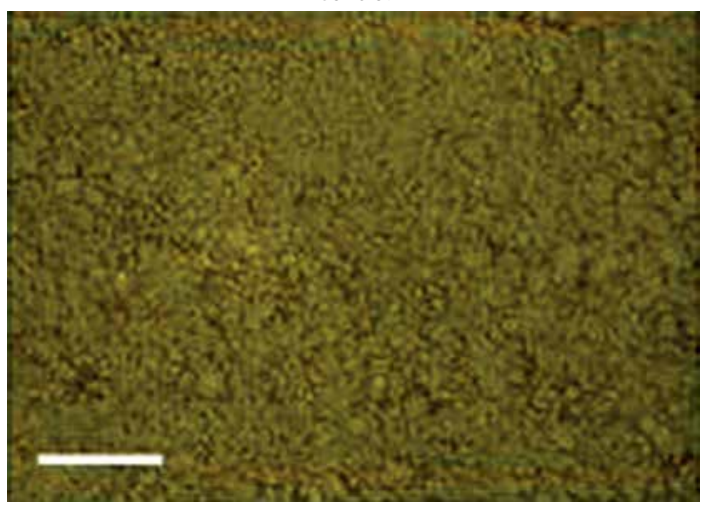

DAPI

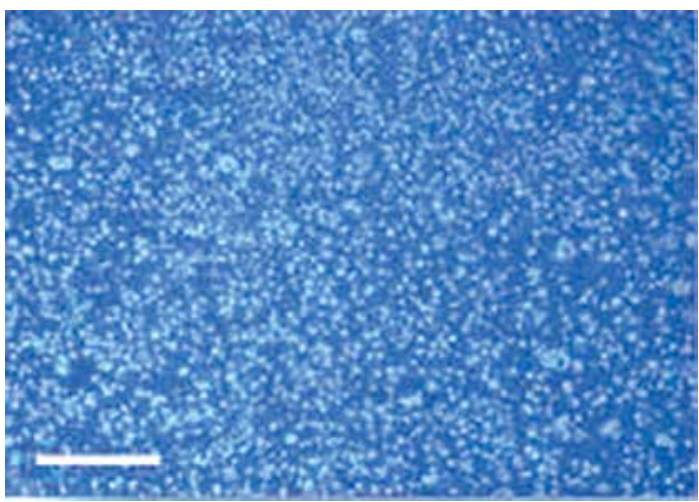

VEGF

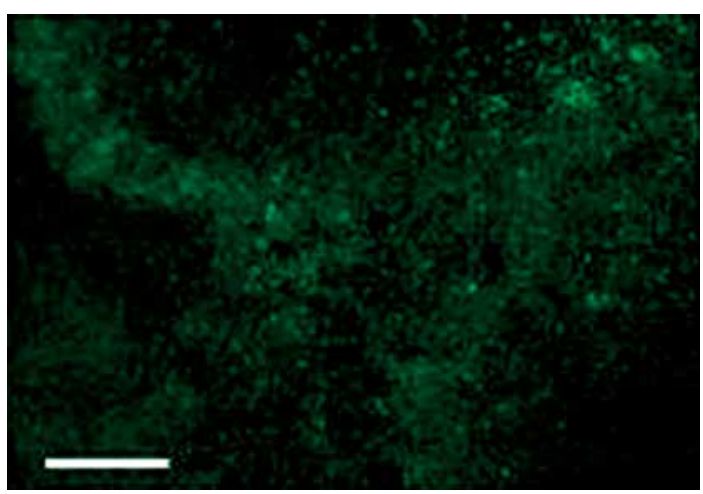

DAPI

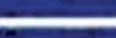

Figure 4. Immunofluorescence analysis of Ki67 and VEGF expression in tumor sections from control and $15 \mathrm{mg} / \mathrm{kg}$ meroxest-treated animals. Nuclei were counterstained with DAPI (blue). Bars: $100 \mu \mathrm{m}$

infiltration is associated with a high tumor grade and high expression of Ki67, both markers of poor prognosis, and decreased survival. In contrast, in $E R^{-}$and $H E R 2^{+}$tumors, infiltration is associated with a good prognosis $[33,34]$. The cell line that
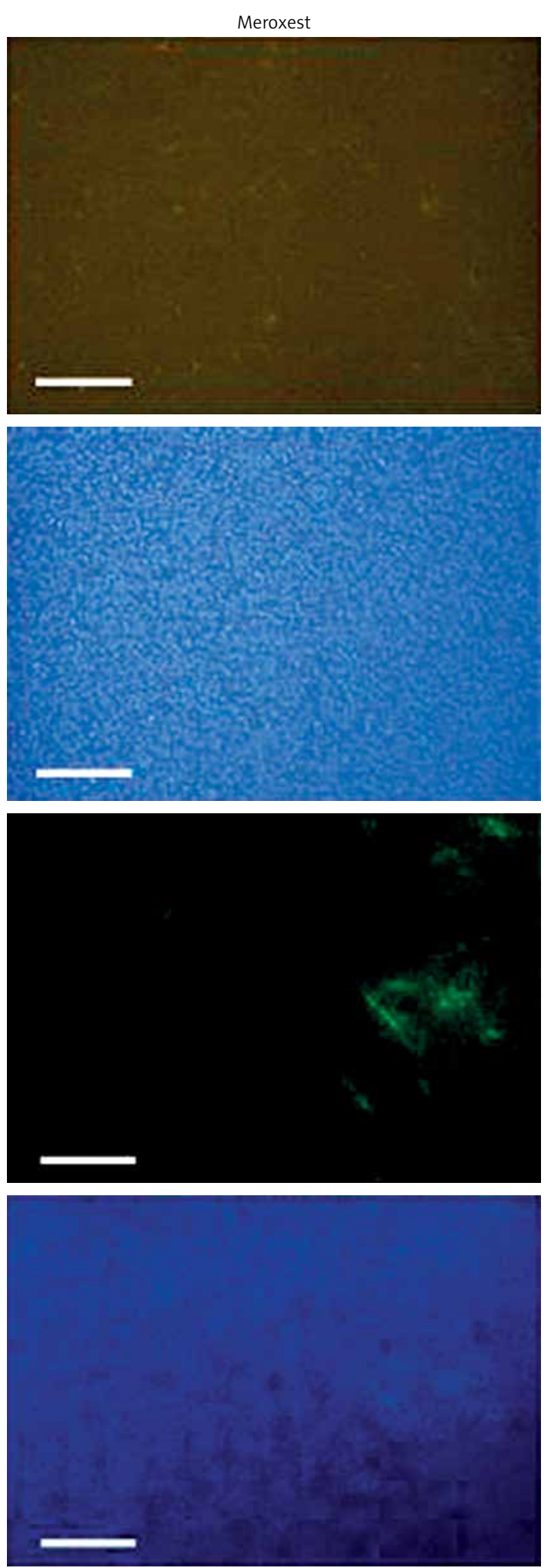

we used for the induction of tumors, E0771, was originally isolated from a spontaneous mouse medullary breast adenocarcinoma. The cells have the phenotype $\mathrm{ER}^{+}$[35]; therefore, and as stated above, meroxest improves the prognosis of treat- 
ed animals by means of a significant reduction in leukocyte infiltration.

Overall, the high cell proliferation rate in tumors is associated with increased aggressiveness and worse prognosis. One method used to assess the state of cell proliferation is the determination of Ki67 marker expression [36]. Ki67 is a nuclear protein that is differentially expressed in the cell cycle, and thus it can only be detected in proliferating cells, not in quiescent cells [37]. There is abundant evidence in favor of the use of Ki67 as a prognostic marker, and that associates increased expression with poor clinical outcome and bad response to chemotherapy [38-41]. In fact, it is a marker employed in the histopathological classification of breast cancer patients. In our study, the tumors of animals treated with meroxest presented a significant decrease in the expression of Ki67 as compared to controls. This effect would indicate a lower proliferative potential of the cells, and a better prognosis for animals, and correlates with our previous results, in which we observed a significant reduction of tumor volume in animals treated with oral meroxest at 5,10 or $15 \mathrm{mg} / \mathrm{kg}$ [22]. Consequently, the volume reduction may be due to the loss of proliferative potential of tumoral cells after treatment with merosesquiterpene.

A very important process for growth, development and progression of a tumor is angiogenesis, which is responsible for new vessel formation. Without vasculature, tumors could not grow beyond 2-3 $\mathrm{mm}$ because they would not receive the necessary nutrients and gases [42]. Therefore, during the growth of primary and metastatic tumors, new vessels are formed. The process of angiogenesis is regulated by a balance established between proangiogenic and antiangiogenic factors $[43,44]$. The most important proangiogenic factor is the VEGF [45]. VEGF, also known as VEGF-A, is a member of the VEGF family, which also includes VEGF-B, VEGF-C, VEGF-D and placental growth factor (PLGF) [46]. VEGF is overexpressed in many human tumors, including breast cancer, which correlates with progression, grade and poor prognosis of the disease [44, 46, 47]. We analyzed the expression of VEGF in our samples and we found over $80 \%$ lower expression in tumors from animals treated with meroxest than control tumors. This effect would indicate a better prognosis for animals, and correlates with our previous results. The lower expression of VEGF could lead to poor development of the tumor vasculature, thereby affecting tumor growth.

Because of the involvement of angiogenesis in tumor progression, antiangiogenic therapy has been presented as a promising strategy in the treatment of disease, and especially in combination with chemotherapy. Among the most commonly used antiangiogenic agents are inhibitors

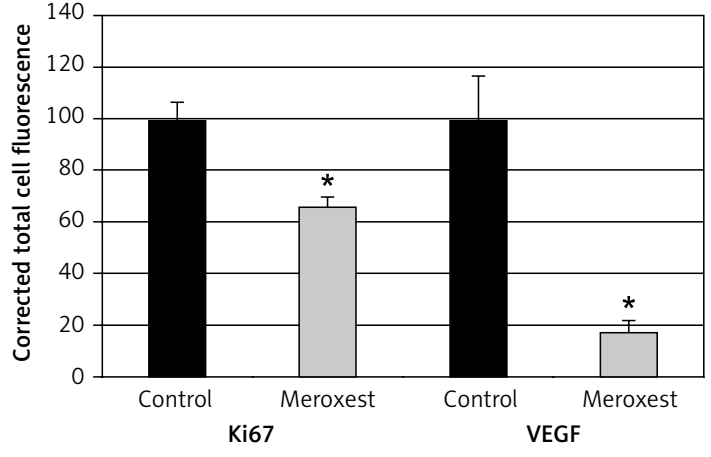

Figure 5. Quantitative analysis of Ki67 and VEGF expression in tumor sections from control and $15 \mathrm{mg} / \mathrm{kg}$ meroxest-treated animals. Total cell fluorescence was calculated using ImageJ software. Mean values and SEM are shown

${ }^{*} P<0.05$ compared with the control group.

of VEGF and its receptors. A leading example is bevacizumab, a VEGF inhibitor that was the first anti-VEGF monoclonal antibody approved by the FDA for the treatment of various types of tumors. Its use in metastatic colon cancer increases survival when given in combination with chemotherapy [48, 49]. However, an important percentage of patients develop resistance to VEGF inhibitors [50-52]. Therefore, it is necessary to discover and/ or develop new antiangiogenic drugs, especially those effective in resistant patients $[53,54]$. In this work, we demonstrate that meroxest leads to a reduction of VEGF expression. Thus, we hypothesize that meroxest may be a useful antiangiogenic drug in the treatment of human breast cancer. In addition, we believe that meroxest may be useful in resistant patients because it interferes with VEGF expression, rather than acting against the expressed protein, which is how most inhibitors act. However, this needs to be clarified in detail.

In conclusion, we believe that allografts of E0771 mouse breast tumor cells in immunocompetent C57BL/6 mice constitute an experimental model of great clinical-translational value in breast cancer. This is because the model provides a natural immune environment, with invasive cells and a high rate of tumor induction. Meroxest improves the prognosis of animals bearing those allografts, since it reduces tumor leukocyte infiltration, and the expression of Ki67 and VEGF markers. Consequently, the merosesquiterpene could become a useful antiangiogenic drug in the treatment of human breast cancer. These results encourage us to deepen the study of meroxest, in order to find more evidence that supports the convenience of its evaluation in a clinical study or trial.

\section{Acknowledgments}

This study was supported by the Regional Government of Andalusia (Project P11-CTS-7651 and 
assistance to the CTS-107 and FQM-348 groups). EC received an FPU pre-doctoral fellowship (Ministry of Education, Culture and Sport), and this article is related to her PhD thesis.

\section{Conflict of interest}

The authors declare no conflict of interest.

\section{References}

1. Piotrowski G, Gawor R, Stasiak A, Gawor Z, Potemski P, Banach M. Cardiac complications associated with trastuzumab in the setting of adjuvant chemotherapy for breast cancer overexpressing human epidermal growth factor receptor type 2 - a prospective study. Arch Med Sci 2012; 8: 227-35.

2. Xu YL, Sun Q, Shan GL, et al. A case-control study on risk factors of breast cancer in China. Arch Med Sci 2012; 8: 303-9.

3. Jemal A, Bray F, Center MM, Ferlay J, Ward E, Forman D. Global cancer statistics. CA Cancer J Clin 2011; 61: 69-90.

4. Mojgan $\mathrm{H}$, Massoud H, Ahmad E. ERCC1 intron 1 was associated with breast cancer risk. Arch Med Sci 2012; 8: 655-8.

5. Dawson SJ, Rueda OM, Aparicio S, Caldas C. A new genome-driven integrated classification of breast cancer and its implications. EMBO J 2013; 32: 617-28.

6. Badowska-Kozakiewicz AM, Sobol M, Patera J, Kozłowski W. Immunohistochemical evaluation of human epidermal growth factor receptor 2 and estrogen and progesterone receptors in invasive breast cancer in women. Arch Med Sci 2013; 9: 466-71.

7. Byler S, Goldgar S, Heerboth S, et al. Genetic and epigenetic aspects of breast cancer progression and therapy. Anticancer Res 2104; 34: 1071-7.

8. Cadoo KA, Fornier MN, Morris PG. Biological subtypes of breast cancer: current concepts and implications for recurrence patterns. Q J Nucl Med Mol Imaging 2013; 57: 312-21.

9. Guiu S, Michiels S, Andre F, et al. Molecular subclasses of breast cancer: how do we define them? The IMPAKT 2012 Working Group Statement. Ann Oncol 2012; 23: 2997-3006.

10. Hashemi-Meshkini A, Keshavarz K, Gharibnaseri Z, et al, Cost-effectiveness analysis review of exemestane in the treatment of primary and advanced breast cancer. Arch Med Sci 2013; 9: 472-8.

11. Lu X, Deng Q, Li H, Suo Z. Altered characteristics of cancer stem/initiating cells in a breast cancer cell line treated with persistent 5-FU chemotherapy. Exp Ther Med 2011; 2: 821-6.

12. Sheri A, Johnston S. New developments and future directions in systemic therapy. Clin Oncol (R Coll Radio) 2013; 25: 117-26.

13. Linjawi S, Khalil W, Hassanane MM, Ahmed ES. Evaluation of the protective effect of Nigella sativa extract and its primary active component thymoquinone against DMBA-induced breast cancer in female rats. Arch Med Sci 2015; 11: 220-9.

14. Robles-Fernández I, Rodríguez-Serrano F, Álvarez PJ, et al. Antitumor properties of natural compounds and related molecules. Recent Pat Anticancer Drug Discov 2013; 8: 203-15.

15. Simpson TJ. Application of isotopic methods to secondary metabolic pathways. In: Biosynthesis. Leeper DFJ,
Vederas PJC (eds). Springer Berlin Heidelberg 1998; $1-48$.

16. Alvarez-Manzaneda EJ, Chahboun R, Barranco Pérez I, Cabrera E, Alvarez E, Alvarez-Manzaneda R. First enantiospecific synthesis of the antitumor marine sponge metabolite (-)-15-oxopuupehenol from (-)-sclareol. Org Lett 2005; 7: 1477-80.

17. Coval SJ, Conover MA, Mierzwa R, et al. Wiedendiol-A and $-\mathrm{B}$, cholesteryl ester transfer protein inhibitors from the marine sponge Xestospongia wiedenmayeri. Bioorg Med Chem Lett 1995; 5: 605-10.

18. Ishii S, Fujii M, Akita H. First syntheses of (-)-tauranin and antibiotic (-)-BE-40644 based on lipase-catalyzed optical resolution of albicanol. Chem Pharm Bull 2009; 57: 1103-6.

19. Talpir R, Rudi A, Kashman Y, Loya Y, Hizi A. Three new sesquiterpene hydroquinones from marine origin. Tetrahedron 1994; 50: 4179-84.

20. Yong KWL, Jankam A, Hooper JNA, Suksamrarn A, Garson MJ. Stereochemical evaluation of sesquiterpene quinones from two sponges of the genus Dactylospongia and the implication for enantioselective processes in marine terpene biosynthesis. Tetrahedron 2008; 64: 6341-8.

21. Bonhoure E, Lauret A, Barnes DJ, et al. Sphingosine kinase-1 is a downstream regulator of imatinib-induced apoptosis in chronic myeloid leukemia cells. Leukemia 2008; 22: 971-9.

22. Carrasco E, Alvarez PJ, Melguizo C, et al. Novel merosesquiterpene exerts a potent antitumor activity against breast cancer cells in vitro and in vivo. Eur J Med Chem 2014; 79: 1-12.

23. Gottwald L, Danilewicz M, Suzin J, et al. Assessment of the argyrophilic nucleolar organizer region area/nucleus ratio in ovarian serous epithelial adenomas, borderline tumors and cancers. Arch Med Sci 2013; 9: 79-85.

24. Sun JZ, Chen C, Jiang G, Tian WQ, Li Y, Sun SR. Quantum dot-based immunofluorescent imaging of Ki67 and identification of prognostic value in HER2-positive (non-luminal) breast cancer. Int J Nanomed 2014; 9: 1339-46.

25. Taneja P, Maglic D, Kai F, et al. Classical and novel prognostic markers for breast cancer and their clinical significance. Clin Med Insights Oncol 2010; 4: 15-34.

26. de Jong M, Maina T. Of mice and humans: are they the same? Implications in cancer translational research. J Nucl Med 2010; 51: 501-4.

27. Kruger JA, Kaplan CD, Luo Y, et al. Characterization of stem cell-like cancer cells in immune-competent mice. Blood 2006; 108: 3906-12.

28. Carrasco E, Alvarez PJ, Prados J, et al. Cancer stem cells and their implication in breast cancer. Eur J Clin Invest 2014; 44: 678-87.

29. Ewens A, Mihich E, Ehrke MJ. Distant metastasis from subcutaneously grown E0771 medullary breast adenocarcinoma. Anticancer Res 2005; 25: 3905-15.

30. Colotta F, Allavena P, Sica A, Garlanda C, Mantovani A. Cancer-related inflammation, the seventh hallmark of cancer: links to genetic instability. Carcinogenesis 2009; 30: 1073-81.

31. Fridman WH, Pagès F, Sautès-Fridman C, Galon J. The immune contexture in human tumours: impact on clinical outcome. Nat Rev Cancer 2012; 12: 298-306.

32. Mohammed ZMA, Going JJ, Edwards J, Elsberger B, Doughty JC, MCMillan DC. The relationship between components of tumour inflammatory cell infiltrate and clinicopathological factors and survival in patients with 
primary operable invasive ductal breast cancer. $\mathrm{Br} J$ Cancer 2012; 107: 864-73.

33. Tsang JY, Hui SW, Ni YB, et al. Lymphocytic infiltrate is associated with favorable biomarkers profile in HER2-overexpressing breast cancers and adverse biomarker profile in ER-positive breast cancers. Breast Cancer Res Treat 2014; 143: 1-9.

34. Tsang JY, Ni YB, Chan SK, et al. Increased lymphocytic infiltration in breast cancer correlated with molecular subtypes and HER2 gene amplification. Histopathology 2013; 62: 963-5.

35. Gu JW, Young E, Patterson SG, et al. Postmenopausal obesity promotes tumor angiogenesis and breast cancer progression in mice. Cancer Biol Ther 2011; 11: 910-7.

36. Herruzo A, Luján S, Moreno J. Cáncer de mama IV. Tratamiento locorregional del cáncer infiltrante de mama. Factores de pronóstico. Tratamiento adyuvante. In: Tratado de ginecología y obstetricia. Cabero i Roura L, Cabrillo Rodríguez E, Bajo Arenas JM (eds.). $2^{\text {nd }}$ ed. Medica Panamericana, Madrid 2012; 860-77.

37. Pathmanathan N, Balleine RL. Ki67 and proliferation in breast cancer. J Clin Pathol 2013; 66: 512-6.

38. Ingolf JB, Russalina M, Simona M, et al. Can Ki-67 play a role in prediction of breast cancer patients' response to neoadjuvant chemotherapy? BioMed Res Int 2014; 2014: 628217.

39. Kim KI, Lee KH, Kim TR, Chun YS, Lee TH, Park HK. Ki-67 as a predictor of response to neoadjuvant chemotherapy in breast cancer patients. J Breast Cancer 2014; 17: 40-6.

40. Urruticoechea A, Smith IE, Dowsett M. Proliferation marker Ki-67 in early breast cancer. J Clin Oncol 2005; 23: 7212-20.

41. Viale G. Pathological work up of the primary tumor: getting the proper information out of it. Breast 2011; 20: S82-6.

42. Folkman J. Tumor angiogenesis: therapeutic implications. N Engl J Med 1971; 285: 1182-6.

43. Hanahan D, Weinberg RA. Hallmarks of cancer: the next generation. Cell 2011; 144: 646-74.

44. Fakhrejahani E, Toi M. Antiangiogenesis therapy for breast cancer: an update and perspectives from clinical trials. Jpn J Clin Oncol 2014; 44: 197-207.

45. Gu JW, Makey KL, Tucker KB, et al. EGCG, a major green tea catechin suppresses breast tumor angiogenesis and growth via inhibiting the activation of HIF-1alpha and NFkappaB, and VEGF expression. Vasc Cell 2013; 5: 9

46. Ferrara N, Gerber HP, LeCouter J. The biology of VEGF and its receptors. Nat Med 2003: 9: 669-76.

47. Ruma IM, Putranto EW, Kondo E, et al. Extract of Cordyceps militaris inhibits angiogenesis and suppresses tumor growth of human malignant melanoma cells. Int J Oncol 2014; 45: 209-18.

48. Lindholm EM, Kristian A, Nalwoga $\mathrm{H}$, et al. Effect of antiangiogenic therapy on tumor growth, vasculature and kinase activity in basal- and luminal-like breast cancer xenografts. Mol Oncol 2012; 6: 418-27.

49. Guo Yt, Ding L, Li Th. [Application of bevacizumab on metastatic breast cancer]. Beijing Da Xue Xue Bao 2012; 44: 708-14.

50. Warin R, Xiao D, Arlotti JA, Bommareddy A, Singh SV. Inhibition of human breast cancer xenograft growth by cruciferous vegetable constituent benzyl isothiocyanate. Mol Carcinog 2010; 49: 500-7.

51. Sharma T, Dhingra R, Singh S, et al. Aflibercept: a novel VEGF targeted agent to explore the future perspectives of anti-angiogenic therapy for the treatment of multiple tumors. Mini Rev Med Chem 2013; 13: 530-40.
52. Potente M, Gerhardt H, Carmeliet P. Basic and therapeutic aspects of angiogenesis. Cell 2011; 146: 873-87.

53. Kerbel RS. Strategies for improving the clinical benefit of antiangiogenic drug based therapies for breast cancer. J Mammary Gland Biol Neoplasia 2012; 17: 229-39.

54. Xynos ID, Tanna N, Patten DK, Palmieri C. Prior exposure to chemotherapy: a marker of sensitivity and selection for antiangiogenic therapy in breast cancer? Expert Rev Anticancer Ther 2014; 14: 163-72. 